\title{
Nanomedicine in organ transplantation and regenerative surgery: an interview with Vijay Gorantla and Jelena Janjic
}

\author{
Jelena M Janjic $c^{\ddagger} 1$ \& Vijay S Gorantla*,‡,2 \\ ${ }^{1}$ Chronic Pain Research Consortium, Graduate School of Pharmaceutical Sciences, Duquesne University School of Pharmacy, 415 \\ Mellon Hall, 600 Forbes Avenue, Pittsburgh, PA 15282, USA \\ ${ }^{2}$ Wake Forest Baptist Medical Center, Director, Vascularized Composite Allotransplantation Program, Wake Forest Institute for \\ Regenerative Medicine, Suite 260, Richard H Dean Biomedical Building, 391 Technology Way, Winston-Salem, NC 27101, USA \\ *Authors for correspondence: Tel.: +1336 713 1494; vgorant|@wakehealth.edu \\ $\ddagger$ Authors contributed equally.
}

Discussing the recently published Special Focus Issue 'Nanomedicine in Organ Transplantation and Regenerative Surgery - Advances, Applications and Future Directions', highlighting the applications of nanomedicines for therapeutic, diagnostic and monitoring strategies in solid organ transplantation as well covering biocompatibility and nanopharmaceutics.

First draft submitted: 21 October 2019; Accepted for publication: 19 November 2019; Published online: 13 December 2019

Keywords: drug delivery $\bullet$ imaging $\bullet$ immunosuppression $\bullet$ nanomedicine $\bullet$ reconstructive surgery $\bullet$ reconstructive transplantation $\bullet$ regenerative medicine $\bullet$ regenerative surgery $\bullet$ transplantation

\section{Lucy Chard}

Q1: Could you tell me how you became involved in researching nanomedicine in organ

transplantation \& regenerative surgery?

Jelena Janjic

I started my career in nanotechnology and nanomedicine in 2006 at Carnegie Mellon University in Pittsburgh (PA, USA). For more than a decade, my lab at Duquesne University (PA, USA) has focused on theranostic technologies, specifically related to pain nanomedicine.

\section{Vijay Gorantla}

I have been involved in clinical and experimental vascularized composite allotransplantation (VCA) almost since its inception in the USA. I was part of the team that performed the first hand transplant in the USA in 1999. Over the past two decades, I have had exposure to both surgical and research aspects of this field and the opportunity to direct the care of several hand transplant recipients. The overarching challenge in VCA is the burden of transplant immunosuppression. We need to rethink and recast transplant medicine in the context of cancer nanomedicine, where advancements have focused on reducing complications and improving outcomes. Novel procedures such as VCA stand to change the lives of millions of people, especially those who are disabled or living with devastating injuries which lack any other standard options.

\section{Lucy Chard}

Q2: How did you first begin working together on this topic?

Jelena Janjic

Dr Gorantla and I began exploring collaborations in applications for nanomedicine in transplantation and regenerative medicine about 3 years ago. Work across our labs span several areas of nanomedicine. Some joint funded projects address nanomedicine applications such as nanoimaging or theranostics in areas such as peripheral nerve injury, nerve regeneration, immune rejection and tissue preservation. 


\section{Lucy Chard \\ Q3: Why is this research an area of unmet need \& what challenges are faced in the field? Jelena Janjic}

We both share similar views on the unmet need, coming from very different perspectives. As a pharmacist and medicinal chemist, nanomedicine offers the unique potential for multifunctionality. For example, we could develop a formulation that can carry single or multiple drugs or agents with different mechanisms of action, which can be precisely delivered to specific cells or tissues of interest and then monitored for activity, treatment response and toxicity. Such theranostic nanomedicine has begun to revolutionize cancer treatment and holds tremendous potential in regenerative medicine or transplantation. Despite such capabilities, nanosystems must be designed with cost and scalability in mind, without which clinical feasibility would be a challenge.

\section{Vijay Gorantla}

If you look at the advancements and developments in transplantation medicine over the past 40 years, only five drugs have been FDA approved in the USA for management of organ transplant rejection. These include cyclosporine (FDA approved in 1983), tacrolimus (FDA approved in 1994), mycophenolate mofetil (FDA approved in 1995), rapamycin (FDA approved in 1999) and most recently belatacept (FDA approved in 2011). Tacrolimus continues to be the major drug in standard organ transplants as well as VCA despite its wide-ranging side effects. In comparison, over the same period, around 200 drugs have been FDA approved for cancer applications versus five drugs in transplantation. Innovation and investment in transplantation medicine has starkly lagged behind oncology. The comparison with oncology is important because it has heavily been in the focus for nanomedicine.

For over 50 years, the overwhelming focus of transplant medicine has been to treat the recipient as a whole to prevent rejection in the donor graft tissues. The question becomes, why do we not use the same approaches in transplantation as in cancer, where treatment is targeted to the cancerous cells or tissues? If we can deliver the anti-rejection drugs predominantly or solely to the graft tissues, we can spare the recipient from the toxicity of the immunosuppression. In essence, we need a paradigm shift in our approach to transplant rejection.

Nanomedicine can deliver ultra-low dose medication to the donor cells or graft tissues and nanoimaging can enable graft surveillance through non-invasive immunomonitoring of donor and recipient cells. Together, we can precisely tune and personalize therapies while minimizing adverse effects as seen with systemic treatments. Beyond improvement in outcomes, the economic justification and savings in healthcare dollars through nanomedicine strategies could be significant.

\section{Lucy Chard}

Q4: Do you think the SFI will have a big impact on the field?

Jelena Janjic

Well, the answer is: definitely. The special focus issue (SFI) offers an integrated multidisciplinary view of the unmet needs and technological advancements in nanomedicine with the specific goal of raising awareness among transplant and regenerative medicine specialists for such technologies.

\section{Vijay Gorantla}

Jelena and I had a vision to bring an issue to the readers that is totally contemporary in terms of topics reviewing therapeutic, diagnostic or monitoring approaches in transplantation or regenerative surgery based on nanomedicine technologies. In addition to promoting peer awareness, the impact we aim for is greater adoption and incorporation of nanomedicine approaches into transplant medicine.

\section{Lucy Chard}

Q5: You were both the authors of the review article 'Pharmaceutical design \& development of perfluorocarbon nanocolloids for oxygen delivery in regenerative medicine' which features in the $\mathrm{SFI}$. Could you give a very brief introduction of this paper \& your rationale for investigating this area? Vijay Gorantla

Tissue preservation is a critical goal in organ transplantation. Despite significant research, funding and investments into hemoglobin substitutes and other artificial oxygen carriers, reliable oxygenation and preservation of tissues 
remains a challenge. In contrast to current oxygenated perfusates, nanocarriers that deliver oxygen into cells can help better optimize oxygenation, facilitating cell metabolism and survival in regeneration or transplantation indications.

Jelena Janjic

The key focus of this article is quality design and manufacturing on a scale of oxygen carrying nanocolloids that are perfectly optimized for cell or tissue oxygenation without the residual toxic side effects of free radicals. These nanocolloids can be loaded with imaging agents to enable non-invasive imaging to monitor ischemia or reperfusion injury events.

\section{Lucy Chard}

Q6: Where do you see the field progressing in the next 5-10 years?

\section{Vijay Gorantla}

We are currently in a moment in transplantation where there is a lot of opportunity and unmet need for technologies or therapies that eliminate the side effects and long-term toxicity of immunosuppressant drugs. Advancements in nanomedicine could help not only better diagnose rejection but also monitor its response to treatment or relapse or resistance to intervention. That is where I think we will see a lot of advancements in the next 5-10 years.

Beyond transplantation, we will also see a lot of advancements in regenerative medicine, especially in the area of bioengineering and tissue engineering. Nanomedicine can be used to generate novel tissues or tissue interfaces as well as bioartificial constructs or 3D tissue constructs and integrating those constructs without the risk of fibrosis, functional compromise or rejection.

\section{Jelena Janjic}

With advancements in pharmaceutical development and quality by design strategies, I am hopeful that nanomedicine will become mainstream and accessible to patients worldwide, especially in areas of regenerative medicine or transplantation where you need long-term treatment with personalization.

\section{Lucy Chard}

Q7: For this issue, you have worked with experts from all over the world. Is this global level of collaboration something that you had in mind when you started the issue?

Jelena Janjic

Innovation in nanomedicine requires multidisciplinary collaboration across disparate and wide-ranging specialties. This is why we engaged subject matter experts from diverse backgrounds to contribute and highlight the latest advancements in nanomedicine and potential applications in transplant or regenerative medicine.

\section{Vijay Gorantla}

Nanomedicine applications in regenerative medicine or transplantation require convergent knowledge. Multiple specialties must come together to create the tools, technologies or therapies that are the most optimal, most safe and most efficacious.

\section{Lucy Chard}

Then to sum up, if you have any final comments that you would like mentioned?

Jelena Janjic

We sincerely hope the special topics address the opportunities and challenges but also highlight the unmet need and the state-of-the-art applications of nanomedicine in transplantation.

\section{Vijay Gorantla}

Although this first special issue is somewhat limited in its focus to organ transplants, reconstructive surgery, tissue engineering and regenerative medicine applications of nanomedicine, we expect to highlight groundbreaking advances in these and other areas in future issues.

\section{Disclaimer}

The opinions expressed in this interview are those of the interviewee and do not necessarily reflect the views of Future Medicine Ltd. 
Financial \& competing interests disclosure

The authors have no relevant affiliations or financial involvement with any organization or entity with a financial interest in or financial conflict with the subject matter or materials discussed in the manuscript. This includes employment, consultancies, honoraria, stock ownership or options, expert testimony, grants or patents received or pending, or royalties. 